\title{
HIGH ENERGY BEAM-BEAM EFFECTS IN CLIC
}

\author{
D. Schulte, CERN, 1211 Geneva 23, Switzerland
}

\begin{abstract}
In order to achieve high luminosity, the Compact Linear Collider (CLIC) has to be operated in the highbeamstrahlung regime at centre-of-mass energies in the few $\mathrm{TeV}$ range. Beam-beam effects for this case are simulated. The dependence of luminosity, luminosity spectrum and background conditions on the different beam parameters is investigated. In particular the effect of beam size, waist shift and offsets are considered; as well as the background due to beamstrahlung and secondary electro-magnetic and hadronic processes.
\end{abstract}

\section{INTRODUCTION}

The beams in future linear colliders must have very small transverse dimensions at the interaction point. This leads to strong transverse electro-magnetic beam fields. In the case of electron positron collision, the field of each beam focuses the other beam. Therefore the luminosity is enhanced. The bending of the trajectories causes the beam particles to emit photons, the beamstrahlung. This effect is comparable to synchrotron radiation and is described by the beamstrahlung parameter $\Upsilon=2 / 3 \cdot E_{c} / E$, where $E_{c}$ is the average critical energy of the emitted photon spectrum and $E$ is the beam energy. The CLIC parameters for different centre-of-mass energies are shown in Table 1 . The main focus of the paper is on the version with a centre-of-mass energy $E_{c m, 0}=3 \mathrm{TeV}$. The designs with $E_{c m, 0} \leq 1 \mathrm{TeV}$ have $\Upsilon \ll 1$, while for higher energies $\Upsilon \gg 1$ is used in order to achieve the required luminosity. As a result, the average energy loss $\delta$ of a beam particle due to beamstrahlung varies from a small correction $\left(3.6 \%\right.$ at $\left.E_{c m, 0}=0.5 \mathrm{TeV}\right)$ to a strong effect $\left(42 \%\right.$ at $\left.E_{c m, 0}=5 \mathrm{TeV}\right)$.

With large $\Upsilon$ the background due to the coherent pair production process is also drastically increased. The additional charge due to this process starts to affect the beambeam interaction itself. This process has therefore been implemented into the program GUINEA-PIG [4].

\section{COHERENT PAIRS}

In a strong external field a photon can turn into an electronpositron pair [1]. This coherent pair creation is strongly suppressed for small values $\kappa$

$$
\kappa=\frac{\hbar \omega}{m c^{2}} \frac{B}{B_{c}}=\frac{\hbar \omega}{E} \Upsilon
$$

where $\hbar \omega$ is the photon energy, $m$ the electron mass, $B$ the magnetic field and $B_{c}=m^{2} c^{2} /(e \hbar)$. The coherent pair production is thus small if $\Upsilon \ll 1$ as it is the case for the colliders at centre-of-mass energies of $1 \mathrm{TeV}$ or below. The number of coherent pairs is given in Table 1. It is negligible at $E_{c m}=0.5 \mathrm{TeV}$. At $E_{c m}=1 \mathrm{TeV}$ it is a possible source of background, while at centre-of-mass energies of $E_{c m} \geq$ $3 \mathrm{TeV}$ it plays a very important role, since $\Upsilon \gg 1$.

The number of particles is, in the latter case, not only important as a background but is not negligible even compared to the number of beam particles. The simulation code had thus to be modified to also include the contribution of these particles to the beam fields.

Since the two particles in the pair have opposite charge and fly in the same direction their electro-magnetic fields neutralise immediately after production. If the particles move in the direction of the electron beam they will not experience a significant force from the electron beam since the electric and magnetic forces cancel. The electron is however focused by the oncoming positron beam while the positron is deflected to the outside. The different charges will thus separate. The equivalent is true for a pair moving in the direction of the positron beam. Effectively the charges of the bunch centres are thus increased.

The effect on the other background sources is not straightforward. While the total luminosity and number of photons is increased the number of high-energy photons is decreased since these have a larger probability of turning into pairs.

\section{RESULTS}

The luminosity spectra for the different parameters in Table 1 are shown in Fig. 1. As expected the higher relative energy loss at higher centre-of-mass energies leads to more degraded spectra, but even at $E_{c m}=5 \mathrm{TeV}$ the fraction of the luminosity with $E_{c m}>0.99 E_{c m, 0}$ remains significant.

The spectrum of produced coherent pair particles is shown in Fig. 2 and their angular distribution after the collision in Fig. 3. The particles are concentrated in small angles, so they would enter a mask that covers the final quadrupoles at the two ends of the detector. However, if they hit material inside these masks-like the final quadrupoles - they would produce secondaries, especially photons and neutrons that could penetrate the mask. Due to the enormous flux of particles this could lead to unacceptable background. It is therefore necessary to have an exit hole of about $10 \mathrm{mrad}$ so that the particles can leave 
Table 1: The beam parameters of CLIC at different centre-of-mass energies. All background numbers are per bunch crossing. The values for $E_{c m} \geq 3 \mathrm{TeV}$ differ from previous ones in [4] due to the inclusion of coherent pair production.

\begin{tabular}{|c|c|c|c|c|c|c|}
\hline centre-of-mass energy & $E_{c m}$ & {$[\mathrm{TeV}]$} & 0.5 & 1 & 3 & 5 \\
repetition frequency & $f_{r e p}$ & {$[\mathrm{~Hz}]$} & 200 & 100 & 75 & 50 \\
bunches per train & $N_{b}$ & & 150 & 150 & 150 & 150 \\
particles per bunch & $N$ & {$\left[10^{9}\right]$} & 4 & 4 & 4 & 4 \\
emittances & $\gamma \epsilon_{x} / \gamma \epsilon_{y}$ & {$[\mu \mathrm{m}]$} & $1.88 / 0.1$ & $1.48 / 0.07$ & $0.6 / 0.01$ & $0.58 / 0.01$ \\
transverse beam sizes & $\sigma_{x}^{*} / \sigma_{y}^{*}$ & {$[\mathrm{~nm}]$} & $196 / 4.52$ & $123 / 2.7$ & $40.4 / 0.58$ & $26.7 / 0.45$ \\
bunch length & $\sigma_{z}$ & {$[\mu \mathrm{m}]$} & 50 & 50 & 30 & 25 \\
beamstrahlung parameter & $\Upsilon$ & & 0.18 & 0.56 & 8.7 & 26.4 \\
\hline luminosity(wo/w coh. pairs) & $\mathcal{L}$ & {$\left[10^{33} \mathrm{~cm}^{-2} \mathrm{~s}^{-1}\right]$} & 6.3 & 13.6 & $133 / 146$ & $186 / 246$ \\
luminosity $\left(E_{c m}>0.99 E_{c m, 0}\right)$ & $\mathcal{L}_{1}$ & {$\left[10^{33} \mathrm{~cm}^{-2} \mathrm{~s}^{-1}\right]$} & 4.5 & 5.1 & 40 & 44.5 \\
average energy loss & $\delta$ & {$[\%]$} & 3.6 & 9.2 & 32 & 42 \\
photons prod. per beam particle & $n_{\gamma}$ & & 0.8 & 1.1 & 2.5 & 4.4 \\
\hline coherent pairs & $N_{c o h}$ & & 3.4 & $2 \cdot 10^{5}$ & $8 \cdot 10^{8}$ & $2.9 \cdot 10^{9}$ \\
pair p. $\left(\theta>0.15, p_{\perp}>20 \mathrm{MeV} / \mathrm{c}\right)$ & $N_{\perp}$ & & 2.9 & 8.0 & 135 & 314 \\
hadronic events $\left(E_{c m}>5 \mathrm{GeV}\right)$ & $N_{H}$ & & 0.022 & 0.15 & 7.8 & 24 \\
minijet pairs $\left(p_{\perp}>10 \mathrm{GeV} / \mathrm{c}\right)$ & $N_{M J}$ & & $2.3 \cdot 10^{-5}$ & $3.9 \cdot 10^{-4}$ & 0.13 & 0.75 \\
\hline
\end{tabular}

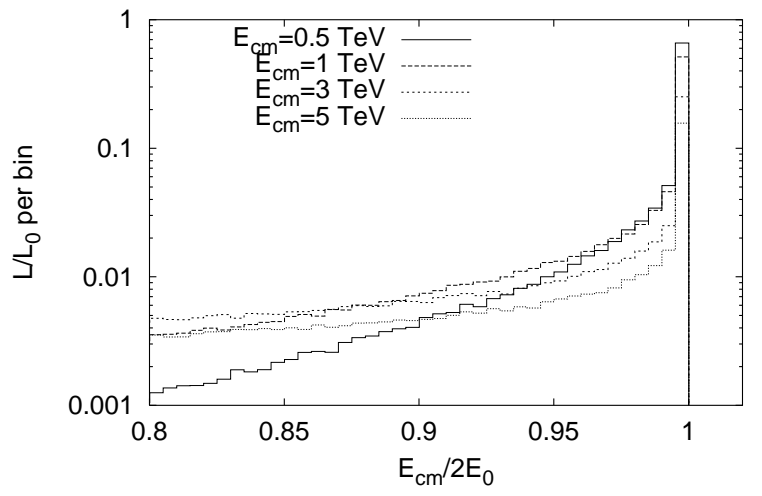

Figure 1: Normalised luminosity spectra for the different centre-of-mass energies.

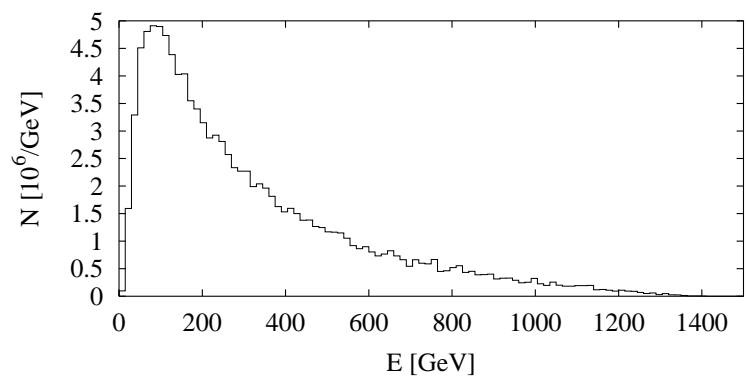

Figure 2: Spectrum of the produced coherent pairs (sum for both beams).

the detector.

\section{INCOHERENT PAIR BACKGROUND}

Electron positron pairs can also be produced via incoherent processes. The main contributions are the BreitWheeler process $\gamma \gamma \rightarrow \mathrm{e}^{+} \mathrm{e}^{-}$, the Bethe-Heitler pro-

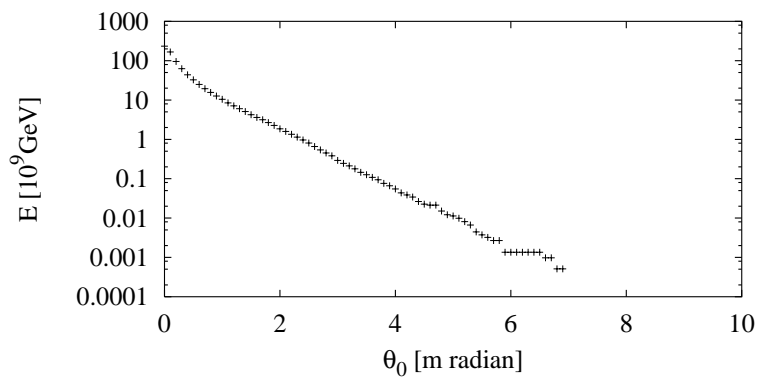

Figure 3: Total energy of the coherent pair particles of one beam with an angle of more then $\theta_{0}$.

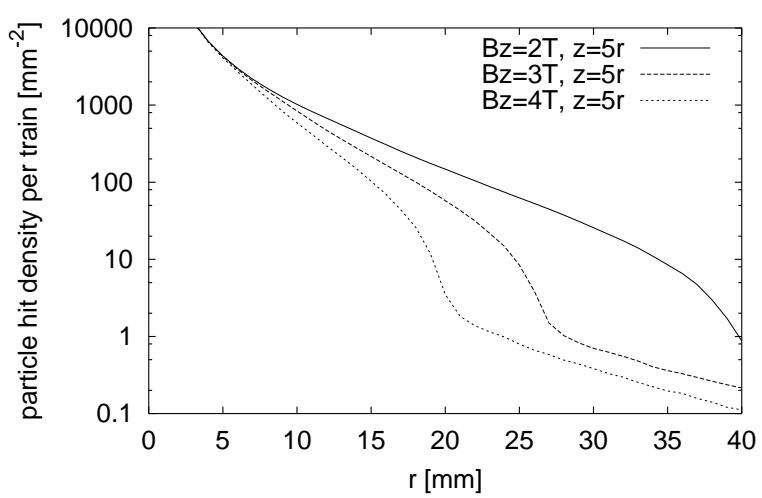

Figure 4: Number of particles that hit a vertex detector for different radii and magnetic fields. The angular coverage was kept constant at $\cos \theta=0.98$.

cess $\mathrm{e} \gamma \rightarrow \mathrm{e}+\mathrm{e}^{+} \mathrm{e}^{-}$and the Landau-Lifshitz process ee $\rightarrow$ ee $+\mathrm{e}^{+} \mathrm{e}^{-}$. The photons are the ones from the beamstrahlung. The last two processes can be calculated replacing the beam particle $\mathrm{e}^{ \pm}$with an equivalent spectrum of virtual photons which are treated as being real. 
The energy of the resulting pair particles is lower than those from the coherent process. While they are deflected the same way by the beam fields, they can have large initial angles. Therefore they can hit a vertex detector and complicate the reconstruction of the trajectories from physics events. Figure 4 shows the number of particles that hit the inner layer of a vertex detector with an opening angle of 200 mrad. Since the crossing angle is not optimised its small effect on the necessary radius is ignored.

\section{HADRONIC BACKGROUND}

Two photon collisions can also result in the production of hadrons. Except for the direct process $\gamma \gamma \rightarrow \mathrm{q} \overline{\mathrm{q}}$, also the one once resolved and twice resolved processes are possible. In these one or both of the photons interact as hadrons. The ansatz for the total cross section follows reference [3]

$$
\sigma_{H}=211 \mathrm{nb} \cdot\left(\frac{s}{\mathrm{GeV}^{2}}\right)^{0.0808}+\sigma_{2} \cdot\left(\frac{s}{\mathrm{GeV}^{2}}\right)^{-0.4525}
$$

Here, $\sigma_{2}=215 \mathrm{nb}$ is expected and $\sigma_{2}=297 \mathrm{nb}$ the most pessimistic value, which is normally used for the calculation of the number of hadronic events comparing different colliders [4]. The calculated number of events per bunch crossing for the $3 \mathrm{TeV}$ design is 7.8 .

An estimate of the hard transverse hadronic background can be found by calculating the number of minijet pairs with a transverse momentum $p_{\perp}>10 \mathrm{GeV} / \mathrm{c}$. For the reference design 0.13 events per bunch crossing are found, see Table 1.

\section{VARIATION OF THE BEAM PARAMETERS}

Without disruption, the optimal positions of the waists of the beam lay in the symmetry plane of the collision $z=$ 0 . With strong disruption, it is advantageous to have the vertical waists of the two beams before this plane [2]. In the case of CLIC this effect is visible, but the difference in luminosity is very small.

Changing the horizontal spot size has a significant impact on the average energy loss of the beam particles during collision. Figure 6 shows the luminosities with a centreof-mass energy larger than a fraction $f=0,0.95,0.99$ of the nominal one. While the luminosity in the high-energy peak of the spectrums depends weakly on the horizontal spot size, the total luminosity does so very strongly. At small $\sigma_{x}$ also the number of coherent pairs increases drastically, their contribution to the total luminosity becomes significant. Depending on the requirements of the experiments, total luminosity can be traded against sharpness of the luminosity spectrum.

\section{CONCLUSION}

At high centre-of-mass energies, CLIC can achieve high luminosity with a spectrum that is still reasonably peaked

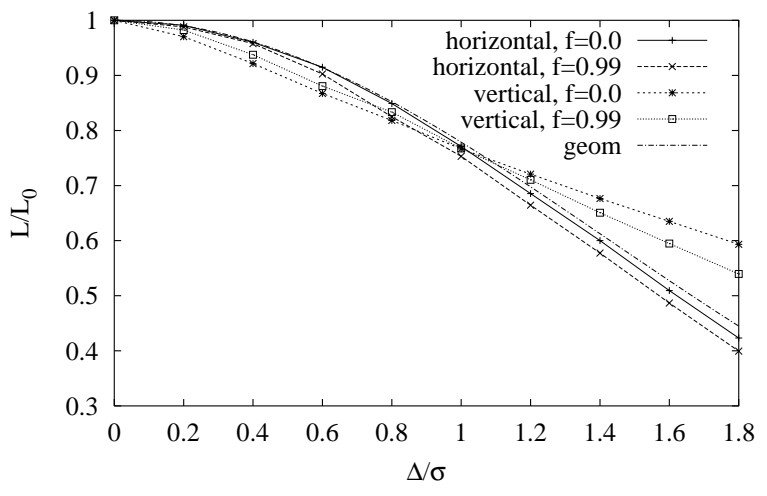

Figure 5: Relative luminosity for different offset normalised to the beam size. The curves correspond to total luminosity, luminosity of collision with $E_{c m}>0.95 E_{c m, 0}$ and $E_{c m}>0.99 E_{c m, 0}$.

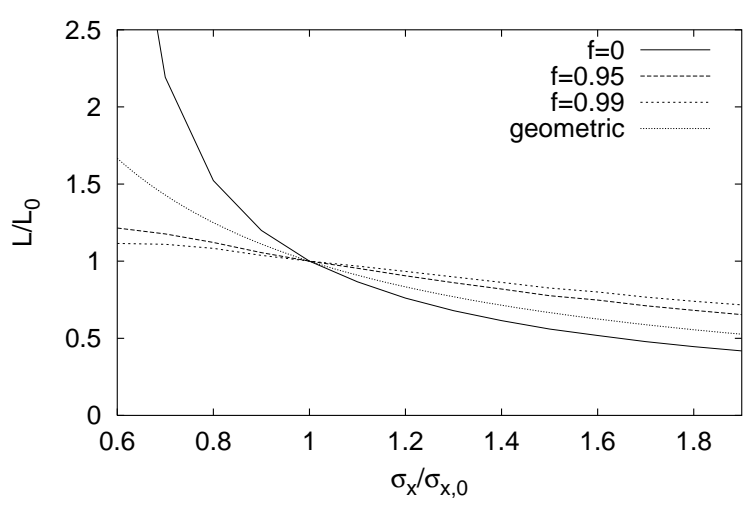

Figure 6: Luminosity for different horizontal spot sizes. The curves correspond to total luminosity, luminosity of collision with $E_{c m}>0.95 E_{c m, 0}$ and $E_{c m}>0.99 E_{c m, 0}$. For comparison the variation of the geometric luminosity is also shown.

at nominal energy. In contrast to lower energy machines, the coherent pair production plays an important role and not only as a background source. It also begins to affect the dynamics of the beam-beam interaction itself. An analysis of the impact the calculated electro-magnetic and hadronic background have on the detector components and on physics experiments remains to be done.

\section{REFERENCES}

[1] P. Chen and K. Yokoya. "Beam-beam phenomena in linear colliders". KEK Prepr. 91-2 (1992).

[2] D. Schulte. "Study of Electromagnetic and Hadronic Background in the Interaction Region of the TESLA Collider". TESLA-97-08 (1996).

[3] G. A. Schuler and T. Sjöstrand. "A scenario for high-energy gamma gamma interactions". Z. Phys. C, 37 (1997) 677688.

[4] D. Schulte. "Beam-beam simulations with GUINEA-PIG". ICAP 1998 and CERN-PS-99-014 (LP). 